\title{
TAVI-in-TAVI - Is this the future?
}

\author{
Szymon Jedrzejczyk, Piotr Scislo, Kajetan Grodecki, \\ Bartosz Rymuza, Janusz Kochman, Zenon Huczek
}

$1^{\text {st }}$ Chair and Department of Cardiology, Medical University of Warsaw, Poland

While an increasing number of younger patients with longer life-expectancy receive transcatheter aortic valve implantation (TAVI), one can assume that patients will outlive their bioprostheses. Thus, repeat interventions after TAVI are expected to rise dramatically. Presented herein are two cases of failed transcatheter heart valves (THV), treated effectively with transcatheter aortic valve-in-valve implantation (TAVI-in-TAVI).

The first, a 75-year-old woman that presented with clinical (exercise-induced dyspnea) and the echocardiographic (aortic valve area [AVA] was $0.75 \mathrm{~cm}^{2}$, AVA index $0.43 \mathrm{~cm}^{2} / \mathrm{m}^{2}$, paravalvular regurgitation) symptoms of bioprosthetic valve failure (BVF), a Sapien XT $23 \mathrm{~mm}$, which was implanted in 2013. Transfemoral TAVI-in-TAVI using the self-expandable Portico $23 \mathrm{~mm}$, THV was performed resulting in a precise implantation (Fig. 1A). Post-operative echocardiography showed an excellent hemodynamic result (AVA $1.62 \mathrm{~cm}^{2}$, AVA index $1.04 \mathrm{~cm}^{2} / \mathrm{m}^{2}$, without para-prosthetic leak), the patient reported symptom improvement and after 7 days was discharged.

The second, a 71-year-old man with clinical symptoms (exercise-induced dyspnea) of heart failure (NYHA III, ejection fraction 27\%) and echocardiographically confirmed severe aortic regurgitation caused mostly by para-prosthetic leak (BVF, CoreValve $29 \mathrm{~mm}$, implanted into the bicuspid valve in 2013). The Heart Team decided to proceed with transfemoral TAVI-in-TAVI using the balloon-expandable Sapien $329 \mathrm{~mm}$ bioprosthesis (Fig. 1B). Optimal implantation was achieved, postoperative echocardiography showed correct function of implanted bioprothesis; the para-prosthetic leak disappeared and gradient was $<10 \mathrm{mmHg}$. The patient was discharged after 6 days.

Taking into consideration the expanding recommendations and rapid growth of TAVI it can be expected that increasingly more BVFs and subsequent TAVI-in-TAVI procedures will occur. Hemodynamics with desired low trans-prosthetic gradient and possible future coronary access should be taken into consideration for optimal clinical effect. TAVI-in-TAVI procedures may also carry an elevated risk of debris embolizing to the brain, however, embolic protection devices can be a potential solution to decrease cerebral embolization and the associated neurological complications.

Conflict of interest: Szymon Jedrzejczyk, Piotr Scislo, Kajetan Grodecki and Bartosz Rymuza declare no conflict of interest. Janusz Kochman is proctor for Abbott and Zenon Huczek is proctor for Medtronic and Abbott.

Address for correspondence: Piotr Scislo, MD, $\mathrm{PhD}, 1^{\text {st }}$ Chair and Department of Cardiology, Medical University of Warsaw, ul. Banacha 1a, 02-097 Warszawa, Poland, tel: +48 2259929 58, fax: +48 22599 19 57, e-mail: piotr.scislo@gmail.com 


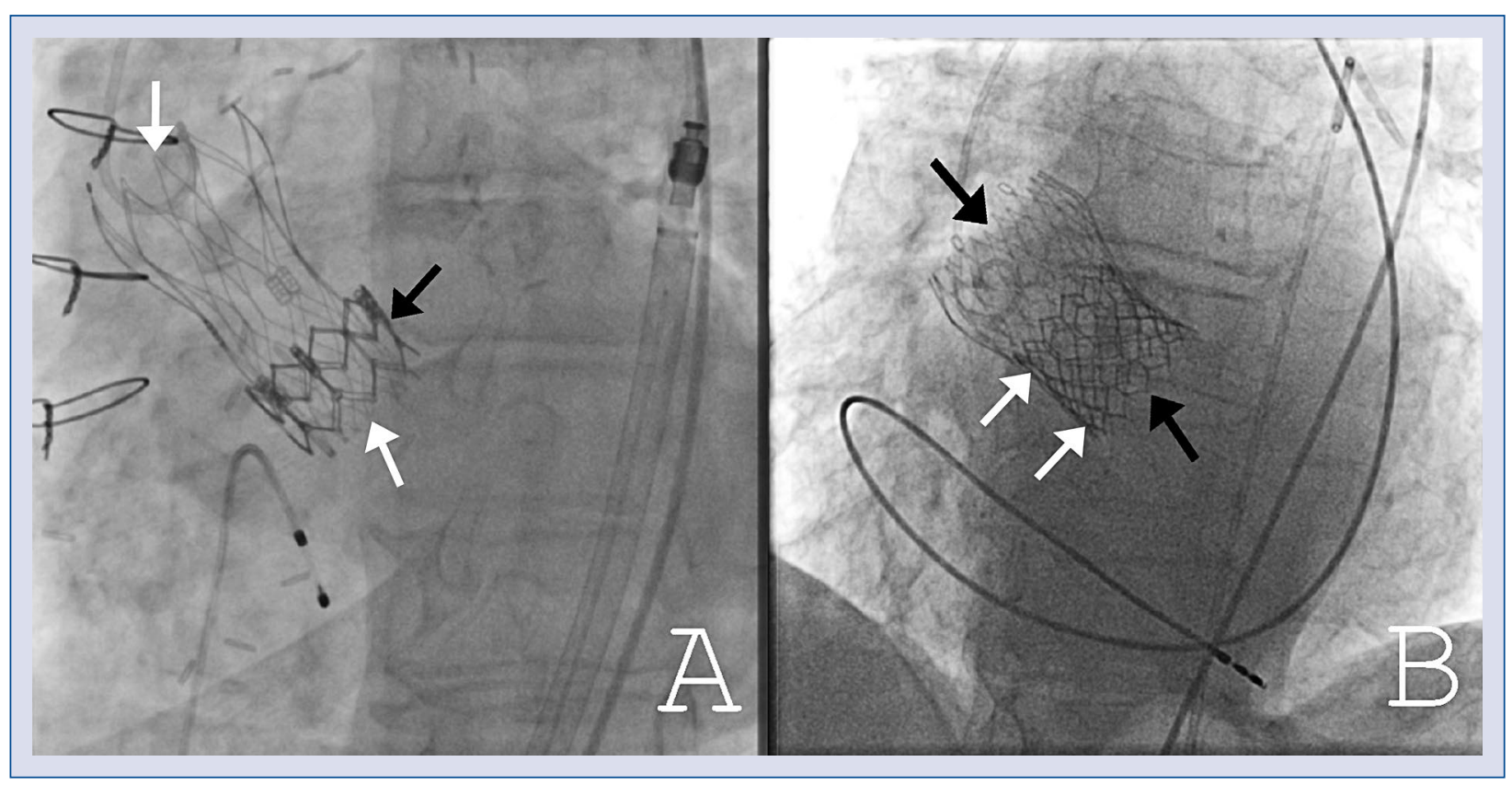

Figure 1. A. Portico $23 \mathrm{~mm}$ (upper and lower edge marked by white arrows) implanted into the failing Sapien XT $23 \mathrm{~mm}$ (marked by black arrow); B. Edwards Sapien $329 \mathrm{~mm}$ (marked by white arrows) implanted into CoreValve $29 \mathrm{~mm}$ (upper and lower edge marked by black arrows). 